\title{
CYCLE BIOLOGIQUE, CROISSANCE ET PRODUCTION DE RHITHROGENA LOYOLAEA NAVAS (EPHEMEROPTERA) DANS UN TORRENT PYRENEEN DE HAUTE MONTAGNE
}

\author{
par P. LAVANDIER ${ }^{1}$
}

\begin{abstract}
Les populations larvaires de Rhithrogena loyolaea ont été étudiées de 1971 à 1973 à 5 altitudes différentes entre la source (altitude : $2350 \mathrm{~m}$, température maximale : $4,5^{\circ} \mathrm{C}$, enneignement : $6-8 \mathrm{mois} / \mathrm{an}$ ) et l'embouchure (altitude : $1850 \mathrm{~m}$, température maximale : $13^{\circ} \mathrm{C}$, enneigement : $4-5$ mois/an) d'un torrent pyrénéen de haute montagne: $1^{\prime}$ Estaragne.
\end{abstract}

Le développement larvaire dure 2 ans au-dessous de $1950 \mathrm{~m}$ et 3 ans au-dessus de $2100 \mathrm{~m}$.

La croissance s'effectue surtout au cours de la période déneigée. Elle est exponentielle en fonction du temps et dépend de la température moyenne du milieu; les taux de croissance instantanés (exprimés en terme de poids) qui varient entre 0,01 et 0,04 jour $^{-1}$ demeurent toutefois faibles à tous les niveaux.

La mortalité a surtout lieu en période déneigée. L'allongement du cycle biologique réduit par cinq le nombre de larves susceptibles d'achever leur développement. La survie larvaire est inférieure à $1 \%$ dans le haut du réseau hydrographique. Les populations sont relativement stables d'une année sur l'autre: leur abondance rend compte des capacités de "charge maximale" du torrent lorsque les conditions sont les plus défavorables.

La production du torrent est en moyenne de $0,6 \mathrm{~g}$ du poids $\mathrm{sec} / \mathrm{m}^{2} / \mathrm{an}$. Les rapports Production/Biomasse moyenne varient entre 2,6 et 3,1.

\section{Life cycle, growth and production of Rhithrogena iloyolaea Navas (Ephemeroptera) in a pyrenean torrential river.}

The larval populations of Rhithrogena loyolaea have been studied from 1971 to 1973 at five different stations between the source (altitude: $2.350 \mathrm{~m}$, maximum temperature: $4.5^{\circ} \mathrm{C}$, snow cover : $6-8$ months per year) and the mouth $(1.850 \mathrm{~m}$, max. temp. : $13{ }^{\circ} \mathrm{C}$, snow cover: $4-5$ months per year) of the river Estaragne, a torrential pyrenean stream.

Larval development took 2 years below $1.950 \mathrm{~m}$ and 3 years above $2.100 \mathrm{~m}$.

Growth occurred chiefly when there was no snow cover, and was an exponential function of temperature, depending on the mean temperature of the environment. The instantaneous growth rate (in terms of weight) varied between 0.01 and 0.04 day $^{-1}$ and always remained low at all stations.

Mortality occurred chiefly when there was no snow cover. The protacted length of the cycle reduced by five times the number of larvae that survived to the end of their development. Larval survival was less than $1 \%$ at the top of the hydrographic system. The populations were relatively stable from one year to the

1. Laboratoire d'Hydrobiologie, ERA 702 du C.N.R.S., Université Paul-Sabatier, 118, route de Narbonne, 31062 Toulouse Cedex. 
next : their abundance reflected the volumes of the "maximum load" of the river when the conditions were most unfavourable.

Mean production was $0.6 \mathrm{~g}$ dry weight per $\mathrm{m}^{2}$ per year and the ratio of production to biomass varied between 2.6 and 3.1 .

Rhithrogena loyolaea est une éphéméroptère Heptageniidae très répandue dans les eaux de montagne où elle constitue une part importante des communautés benthiques. Les caractéristiques du développement des œufs ont été établies récemment (Humpesch et Elliott 1980). Le développement post-embryonnaire qui semble variable d'un milieu à un autre (Landa 1968, Sowa 1975) est en revanche, mal connu de même que la croissance larvaire et la dynamique des populations.

Dans ce travail, la densité des populations, le cycle biologique, la croissance et la production larvaires sont étudiés à différentes altitudes d'un torrent de haute montagne. Le gradient des conditions de milieu lié à l'élévation en altitude permet de mettre en évidence les facultés d'adaptation de l'espèce dans un milieu torrenticole extrême et d'appréhender l'influence des principaux facteurs écologiques sur la répartition et la dynamique du peuplement.

\section{1. - MILIEU — MATERIEL — METHODE}

Situé dans les Pyrénées centrales, le vallon d'Estaragne a été décrit en détail dans un travail antérieur (Lavandier 1974). Le torrent est issu d'un névé vers $2380 \mathrm{~m}$ d'altitude; il se jette dans le lac d'Orédon à $1850 \mathrm{~m}$ après un cours Sud-Nord de $2,7 \mathrm{~km}$ environ. Il présente deux importantes ruptures de pente: dans la partie amont entre 2350 et $2280 \mathrm{~m}$ d'altitude et dans la partie médiane au niveau d'un ressaut glaciaire entre 2000 et $1930 \mathrm{~m}$ d'altitude. Le lit, rocheux, est couvert de neige 4 à 8 mois par an selon l'altitude. Sous la neige les tempéra. tures sont partout voisines de $0^{\circ} \mathrm{C}$; au cours de l'été elles dépassent $4^{\circ} \mathrm{C}$ en août près de la source et $13^{\circ} \mathrm{C}$ à l'embouchure où les amplitudes quotidiennes peuvent atteindre $9^{\circ} \mathrm{C}$. Les crues sont violentes à la fonte des neiges et lors des orages, avec des vitesses de courant supérieures à $2,5 \mathrm{~m} / \mathrm{s}$. Les cinq localités étudiées sont situées sur le torrent et correspondent aux stations A. B. R. C. D. dans les descriptions antérieures du réseau hydrographique; leur localisation et le régime des températures à leur niveau sont rappelés dans la figure 1 .

Le torrent a été prospecté de 1971 à 1973 durant la période d'accessibilité du vallon (juin-novembre). Les adultes et les subimagos étaient capturés chaque semaine par chasse, pièges à émergence ou dans des filets de dérive. Les larves étaient recueillies à l'aide de filet de Surber 

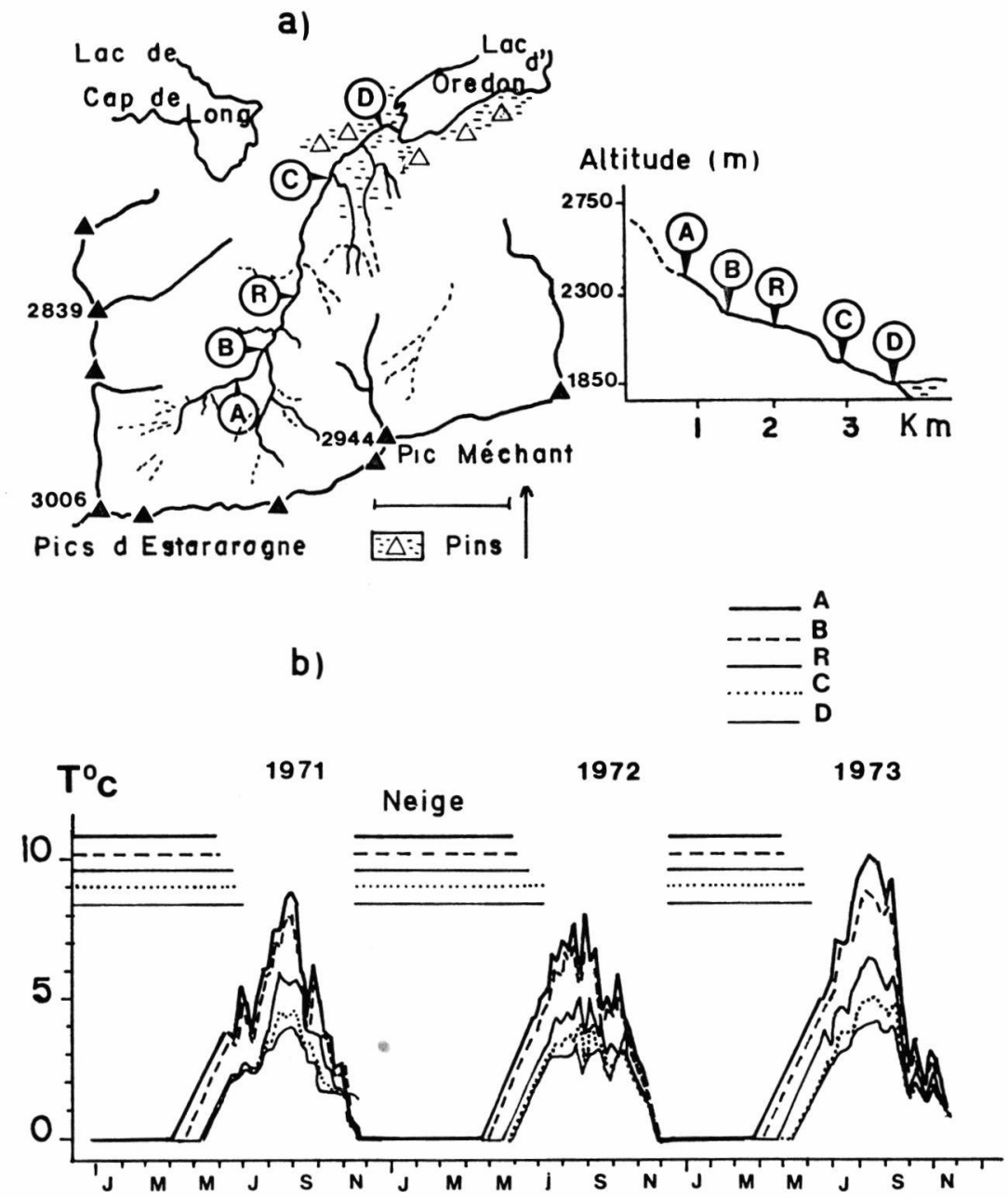

FIG. 1. - a) Réseau hydrographique de l'Estaragne et stations d'étude; b) Evolution saisonnière des températures moyennes hebdomadaires (cf. Lavandier 1974). Aux stations B (en 1971) et C (en 1973), les valeurs sont déduites des températures relevées aux localités voisines.

de vide de maille de 0,1 et $0,15 \mathrm{~mm}$. A la station $\mathrm{R}$, dix à quinze relevés couvrant 1 à $1,5 \mathrm{~m}^{2}$ étaient réalisés dans les divers habitats toutes les trois semaines en 1971, tous les mois en 1972 et 1973, aux autres stations quatre à six relevés (sur $0,5 \mathrm{~m}^{2}$ environ) étaient faits chaque mois.

Les prélèvements étaient formolés sur le terrain et la faune triée au laboratoire, conservée en alcool. 


\section{2. - REPARTITION}

Dans le réseau hydrographique, Rhithrogena loyolaea peuple surtout le torrent. Son abondance est maximale dans la partie médiane du cours où la moyenne des effectifs mensuels varie entre 2000 (à $2190 \mathrm{~m}$ d'altitude) et 2900 individus $/ \mathrm{m}^{2}$ (à $2150 \mathrm{~m}$ d'altitude); l'abondance diminue vers l'aval (600 individus $/ \mathrm{m}^{2}$ à 1920,220 individus $/ \mathrm{m}^{2}$ à l'embouchure) et vers l'amont ( 900 individus $/ \mathrm{m}^{2}$ près de la source) où seules quelques larves parviennent au dernier stade.

Les jeunes larves peuplent tous les habitats avec toutefois une préférence pour les zones calmes de sable et de graviers ; les larves âgées sont plus rhéophiles et colonisent davantage les galets, les blocs rocheux et les cascades. Dans les affluents au-dessous de $2200 \mathrm{~m}$, Rhithrogena loyolaea n'est présente qu'à l'état de larves jeunes.

\section{3. - CYCLE BIOLOGIQUE}

Les cycles biologiques sont déduits de la structure des populations à chaque date d'échantillonnage. Les générations qui coexistent dans le milieu sont affectées des indices $0^{+}, 1^{+}, 2^{+}, 3^{+}$selon qu'elles ont passé $0,1,2$ ou 3 hivers. La largeur (l) de la capsule céphalique des larves est utilisée comme critère de taille; on peut en déduire la longueur $(\mathrm{L})$ des larves par la relation : $\mathrm{L}(\mathrm{mm})=3,31 \mathrm{l}(\mathrm{mm})$ $-0,055$. En fin de cycle, la longueur des fourreaux alaires antérieurs permet de reconnaître le stade de développement des individus (fig. 2).

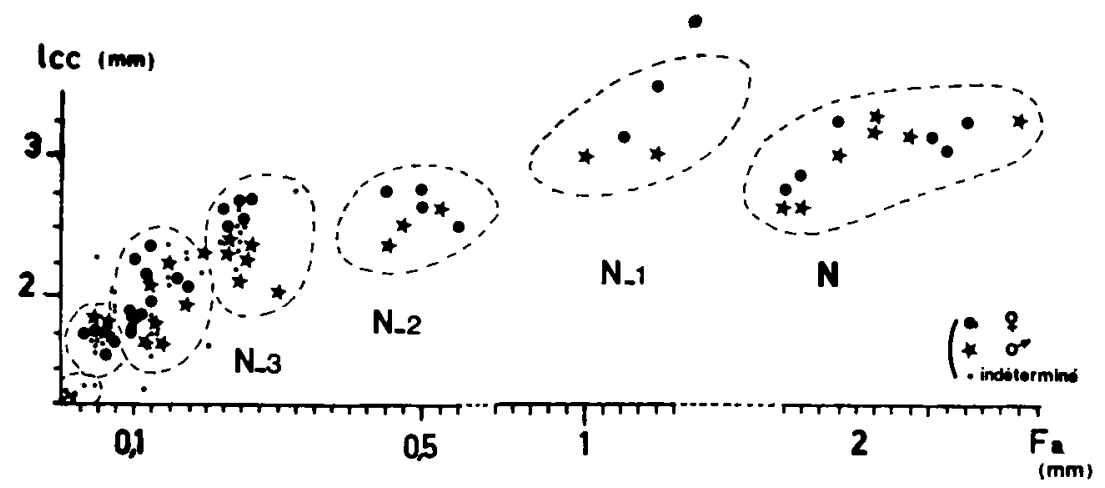

Fig. 2. - Différenciation des derniers stades larvaires de Rhithrogena loyolaea. lcc : largeur des capsules céphaliques; $\mathrm{Fa}$ : longueur des fourreaux alaires antérieurs ; $\mathbf{N}$ : dernier stade larvaire ; $\mathbf{N}-1$ : avant-dernier stade larvaire...

Au-dessous du ressaut glaciaire, deux ou trois générations coexistent en permanence : elles caractérisent un développement semi-voltin (fig. 


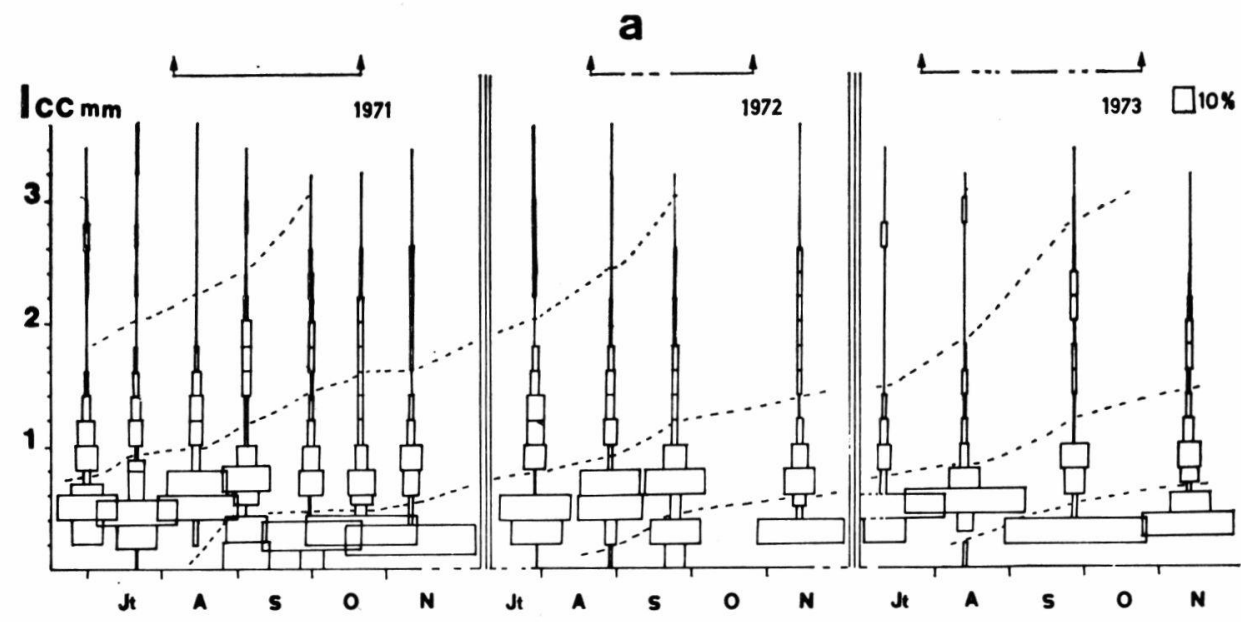

b

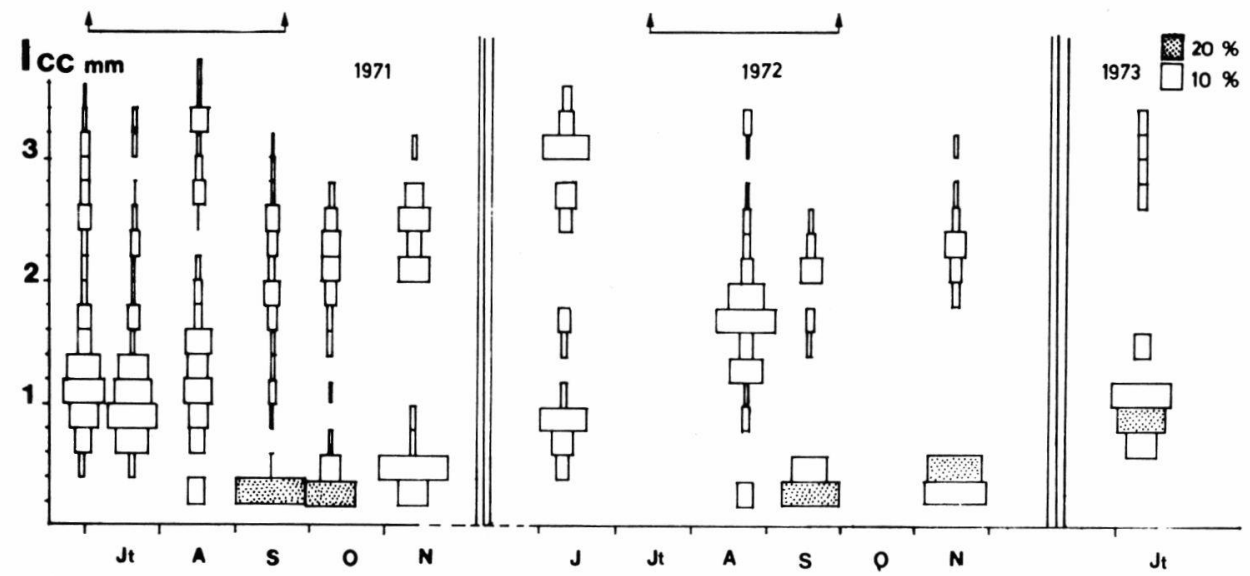

Fıc. 3. - Développement larvaire de Rhithrogena loyolaea. a) Dans le cours supérieur du torrent (station $\mathrm{R}$, altitude : $2150 \mathrm{~m}$ ) ; b) Dans le cours inférieur (station $\mathrm{D}$, altitude : $1850 \mathrm{~m}$ ) ; lcc : largeur des capsules céphaliques.

3 a). La période d'éclosion déduite de la période d'apparition des plus petits individus capturés $\left(0^{+}\right)$débute en août et se prolonge jusqu'à l'hiver. Le développement larvairle, lent sous la neige et durant la crue printanière, s'accélère en été puis faiblit légèrement en automne. Les larves $\left(1^{+}\right)$passent un deuxième hiver de vie ralentie et les premiers imagos s'envolent dès le mois de juillet suivant. Les différentes phases du cycle sont légèrement plus précoces à $1850 \mathrm{~m}$ (station D) qu'à 1920 m d'altitude (station C). 
Au-dessus du ressaut glaciaire, trois ou quatre générations s'individualisent à chaque date d'échantillonnage (fig. $3 \mathrm{~b}$ ). Le début des éclosions se retarde de juillet à octobre avec l'élévation en altitude; elles se poursuivent jusqu'en fin d'année et de nombreuses larvules subsistent encore au printemps suivant. Le développement a essentiellement lieu durant les mois les plus chauds de la phase déneigée; il se stabilise en hiver et au printemps et faiblit dès octobre. A l'entrée du deuxième hiver, les larves $\left(1^{+}\right)$n'ont pas encore d'ébauches de fourreaux alaires; ils apparaissent et grandissent au cours de l'été suivant, mais le cycle ne s'achève qu'après la crue nivale consécutive à un troisième hiver de vie aquatique. L'envol des imagos débute fin juillet vers $2150 \mathrm{~m}$, en août vers $2190 \mathrm{~m}$, en septembre près de la source $(2370 \mathrm{~m})$. A ce niveau seul un petit nombre de larves paraît achever son cycle biologique : en trois années de prospection, deux larves-nymphes et trois subimagos seulement ont été capturés; en revanche, une vingtaine d'imagos femelles venant de pondre ont été recueillies par dérive. Cette proportion qui diffère totalement de celles observées sur le reste du réseau hydrographique où pour un imago, neuf subimagos au moins étaient capturés montre l'importance des migrations imaginales. Dans le haut du réseau hydrographique, les femelles issues d'aval assurent chaque année par leur ponte l'essentiel de la nouvelle génération.

\section{4. - CROISSANCE PONDERALE}

La croissance est estimée d'après l'évolution pondérale des larves en fonction du temps. Dans chaque cohorte, le poids individuel moyen est calculé à partir du poids des effectifs de chaque classe de taille. Cette méthode réduit la sousestimation qui résulte de l'utilisation de la taille moyenne pour évaluer le poids à l'aide d'une relation taille - poids de forme exponentielle (Hamilton 1969). Les Iarves sont pesées après déssèchement à l'étuve à $60^{\circ} \mathrm{C}$ pendant 24 heures. Le poids sec moyen (PS $\mathrm{mg}$ ) et la largeur $(1 \mathrm{~mm})$ des capsules céphaliques des larves sont liés par la relation $\log P s=2,97 \log 1-1,716$.

Durant les périodes déneigées successives, le poids larvaire moyen dessine en fonction du temps une série de courbes qui peuvent être assimilées à des portions de courbes exponentielles (fig. 4) et la relation entre le poids individuel moyen des larves de chaque cohorte et le temps peut s'écrire: $\mathrm{Pt}=\mathrm{Po} \mathrm{e}^{\mathrm{KT}}$ avec $\mathrm{Pt}=$ poids au temps $\mathrm{t}$, Po $=$ poids au temps to, $\mathrm{T}$ en jours, $\mathrm{K}$ étant le taux de croissance instantané. Ce modèle classique de croissance qui rend bien compte des observations en période déneigée a également été appliqué à la

Fig. 4. - Evolution des effectifs larvaires $\left(\mathrm{N} / \mathrm{m}^{2}\right)$ et croissance du poids individuel moyen (Poids sec: $\mathrm{P}$ en $\mathrm{mg}$ ) des larves de chaque cohorte aux différentes stations. 
(7) CROISSANCE ET PRODUCTION DE RHITHROGENA LOYOLAEA
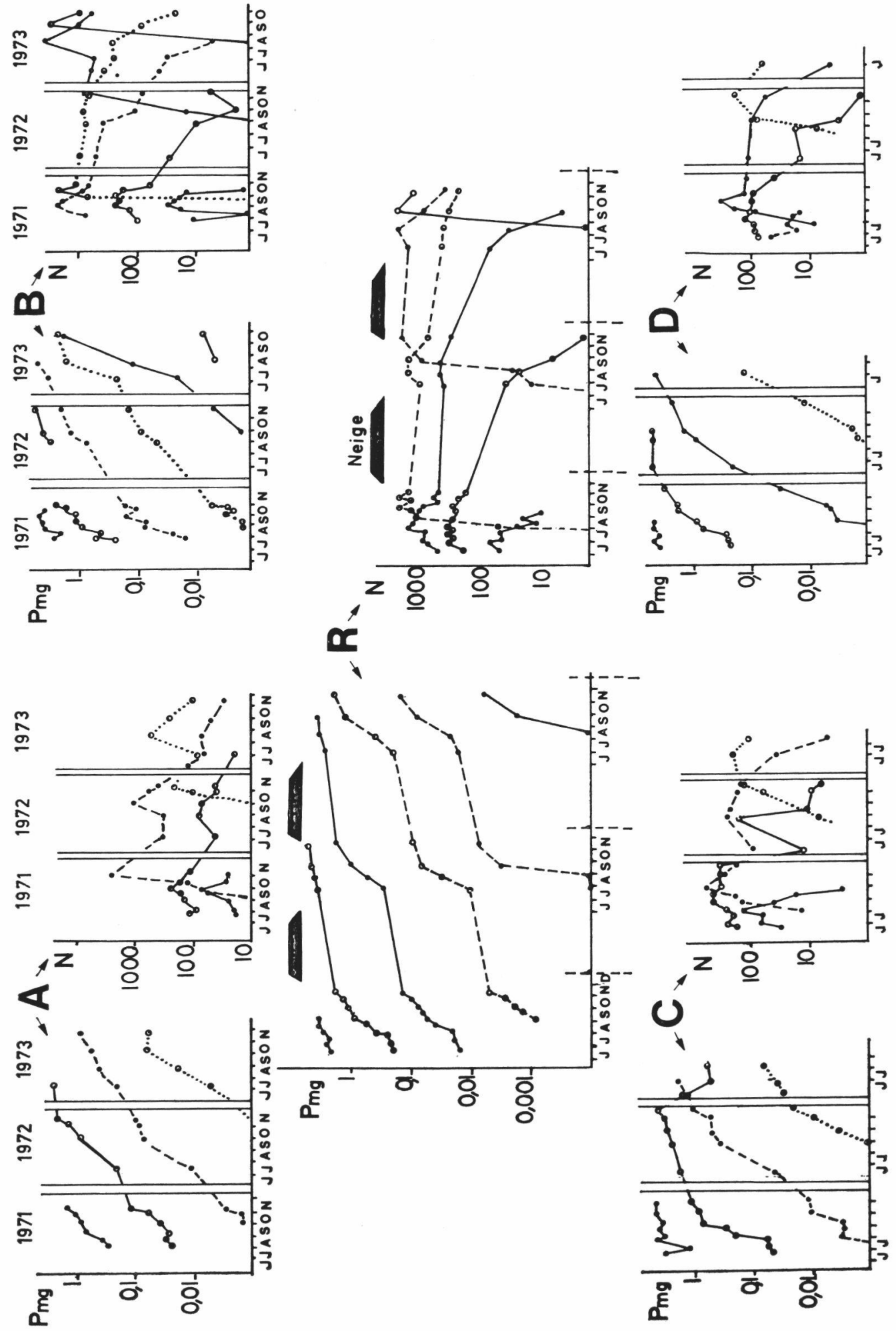
P. LAVANDIER

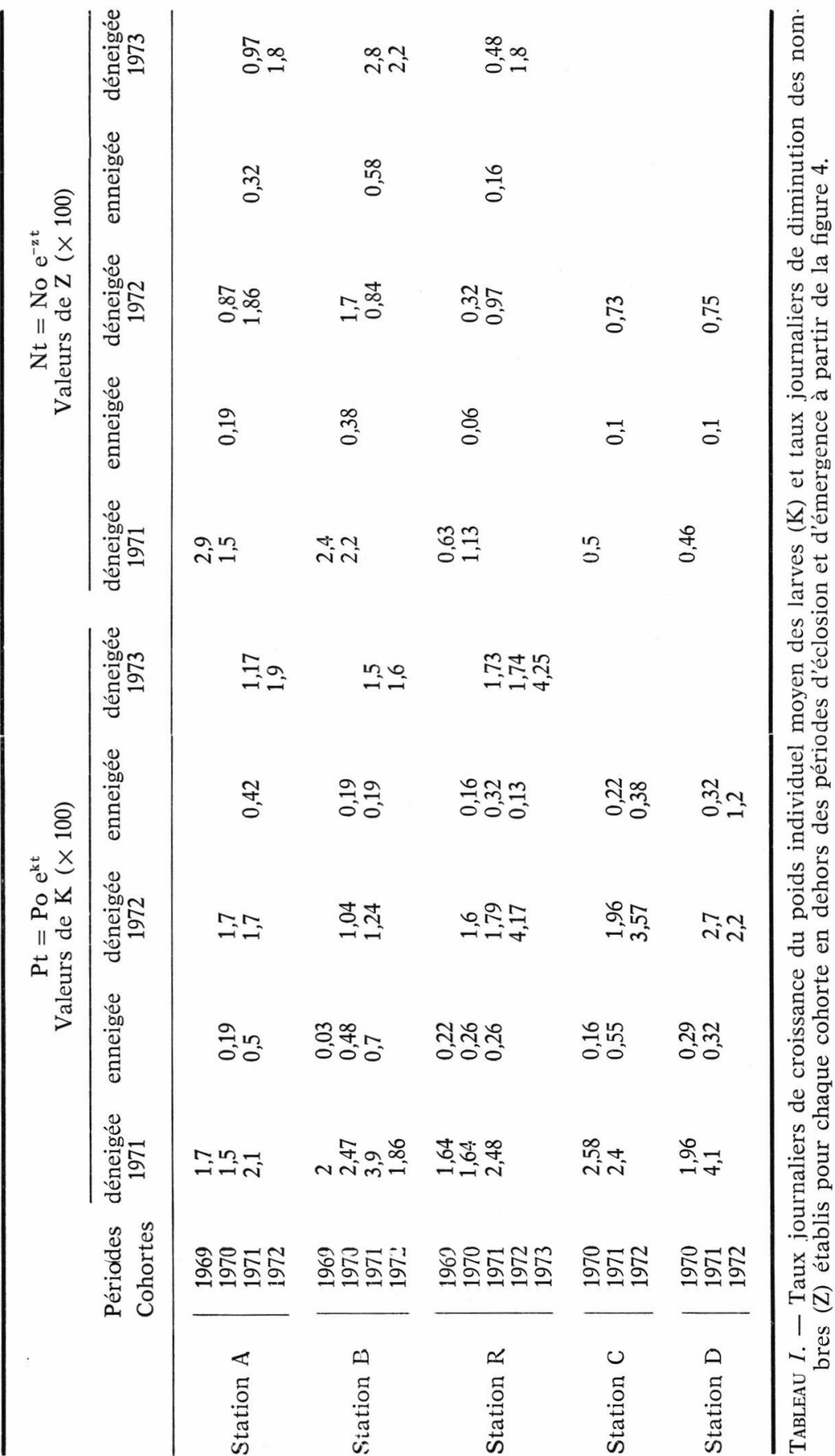


période hivernale comprise entre le dernier relevé d'automne et le premier relevé du printemps suivant.

Sous la neige, les taux de croissance instantanés sont faibles $(\overline{\mathrm{K}}=$ $0,0037 \pm 0,0029$ ) et du même ordre dans tout le réseau hydrographique. Les différences observées annuellement ou entre stations dépendent essentiellement de la date du premier échantillonnage printanier plus ou moins éloigné de la reprise de croissance.

Après la fonte des neiges, les taux de croissance sont plus élevés; ils diminuent au cours du développement et sont généralement comparables d'une cohorte à la suivante pour des périodes homologues de la vie larvaire (Tableau I).

Les taux de croissance instantanés $(K)$ de chaque cohorte calculés en dehors des périodes d'éclosion et d'émergence dépendent de la température moyenne du milieu (fig. 5). La liaison est hautement significative pour toutes les classes d'âge sous la forme $\mathrm{K}=\mathrm{aT}+\mathrm{b}$; pour les larves $0^{+}$et $1^{+}$elle est toutefois légèrement plus forte lorsque la température est exprimée en logarithmes (Tableau II). La pente de la régression obtenue pour les larves néonates est significativement plus forte que les pentes établies pour les autres cohortes. Les pentes relatives aux générations âgées de un et deux ans ne présentent pas de différences significatives.

Ainsi, l'augmentation de température a une influence d'autant plus grande sur la croissance que la température initiale est basse et que les larves sont jeunes.

\begin{tabular}{|c|c|c|c|c|c|c|c|}
\hline & \multicolumn{3}{|c|}{$\mathrm{K}=\mathrm{aT}+\mathrm{b}$} & \multirow[b]{2}{*}{$N$} & \multicolumn{3}{|c|}{$\mathrm{K}=\mathrm{a} \log \mathrm{T}+\mathrm{b}$} \\
\hline & $\mathbf{a}$ & $b$ & $\mathbf{r}$ & & $a$ & b & $\mathrm{r}$ \\
\hline Cohorte $\mathrm{C}^{+}$ & $\begin{array}{r}0,00927 \\
\pm 0,00315\end{array}$ & $\begin{aligned} & 0,00118 \\
\pm & 0,017\end{aligned}$ & 0,84 & 17 & $\begin{array}{r}0,0194 \\
\pm 0,0056\end{array}$ & $\begin{array}{c}0,01 \\
\pm 0,016\end{array}$ & 0,87 \\
\hline Cohorte $1^{+}$ & $\begin{aligned} & 0,00466 \\
\pm & 0,001\end{aligned}$ & $\begin{array}{r}0,0006 \\
\pm 0,0086\end{array}$ & 0,88 & 23 & $\begin{aligned} & 0,0095 \\
\pm & 0,002\end{aligned}$ & $\begin{aligned} & 0,00633 \\
\pm & 0,0081\end{aligned}$ & 0,90 \\
\hline Cohorte $2^{+}$ & $\begin{aligned} & 0,00584 \\
\pm & 0,0013\end{aligned}$ & $\begin{array}{l}-0,00295 \\
\pm 0,0058\end{array}$ & 0,93 & 13 & $\begin{array}{r}0,0103 \\
\pm 0,0026\end{array}$ & $\begin{aligned} & 0,00396 \\
\pm & 0,0066\end{aligned}$ & 0,92 \\
\hline
\end{tabular}

T'ableau II. - Relation entre le taux de croissance (K) et la température moyenne du milieu ( $\mathrm{T}$ ou Log. T). Valeurs de a et de b (avec limites de confiance à $95 \%$ ). Les valeurs du coefficient de corrélation sont hautement significatives. 


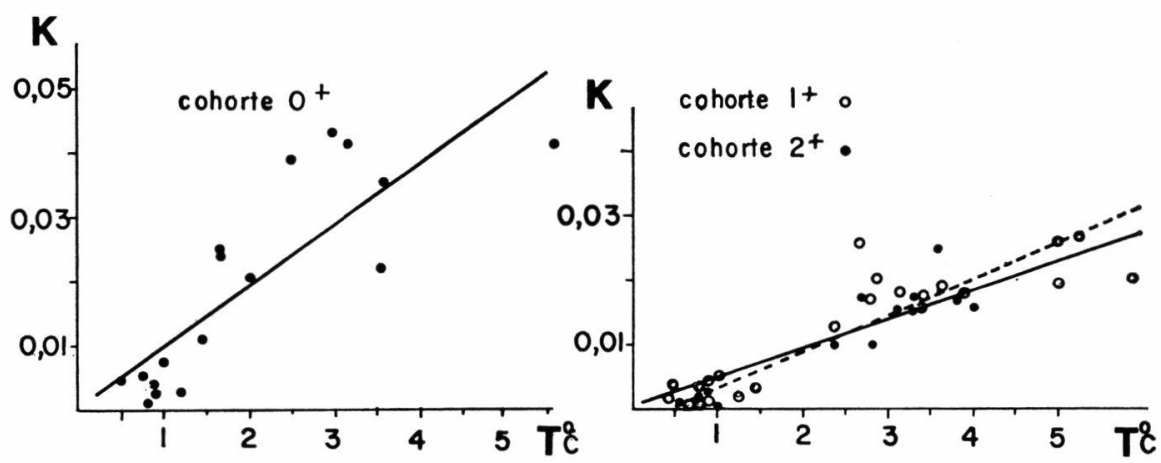

Fig. 5. - Relation entre le taux journalier de croissance instantanée $\mathrm{K}$ des différentes cohortes $\left(\mathrm{CO}^{+}, \mathrm{C1}^{+}, \mathrm{C2}^{+}\right)$et la température moyenne, $\mathrm{T}$, du milieu.

\section{5. - EVOLUTION NUMERIQUE}

A chaque échantillonnage, un nombre moyen d'individus par $\mathrm{m}^{2}$ est calculé pour chaque cohorte. Compte tenu des caractéristiques des échantillons, les limites de confiance à $95 \%$ de la moyenne peuvent être estimées en appliquant à la moyenne arithmétique un facteur $\mathrm{F}=\mathrm{t} \mathrm{x}$ erreur standard obtenu après transformation logarithmique des données (Elliott 1978, p. 90 et suivantes). A la station R, $\mathrm{F}$ est en moyenne de 1,6 (génération $0^{+}$), de 1,48 (génération $1^{+}$), de 1,35 (génération $2^{+}$) et de 1,3 pour la génération $3^{+}$. Aux autres stations où l'échantillonnage a été moins fourni, les valeurs de $\mathrm{F}$ sont en général plus élevées de 0,2 - pour les larves néonates - à 0,6 unités - pour les larves âgées - Les limites de confiance sont légèrement plus étroites à l'étiage que lors des crues.

En début de cycle biologique, le nombre de larvules augmente avec les éclosions jusqu'à ce que la mortalité l'emporte sur le recrutement. Enfin de cycle biologique, la rapide disparition des larves est due aux émergences. Entre ces deux périodes et à tous les niveaux, l'évolution numérique se caractérise après chaque hiver par une courbe en cloche dont le mode correspond à l'été (fig. 4).

La partie ascendante de la courbe résulte d'une recolonisation progressive du lit du torrent lors de la décrue (Lavandier 1979 b). A la fonte des neiges en effet, la faune gagne des habitats refuges à l'abri du courant et devient de ce fait difficile à capturer. Elle réapparaît peu à peu lorsque les débits se réduisent et les effectifs augmentent. L'effectif maximum, qui marque l'achèvement apparent de la migration s'observe lorsque la vitesse du courant tombe à $50 \mathrm{~cm} / \mathrm{s}$ environ. La mortalité relative à cette période, déduite de la comparaison des derniers relevés d'automne et des premiers relevés de printemps est faible et du même ordre dans tout le torrent: les trois-quarts des individus survivent aux conditions hivernales et printanières.

La partie descendante de la courbe qui traduit la mortalité larvaire ( + dérive) peut être assimilée à des portions de courbes exponentielles 
décroissantes (NT $=$ Noe-zT avec $\mathrm{T}$ en jours, NT et No $=$ effectifs aux temps $\mathrm{T}$ et To, $\mathrm{Z}$ étant le taux instantané de diminution des nombres). Les taux de diminution des nombres $\mathrm{Z}$ ont été calculés en dehors des périodes d'éclosion et d'émergence (Tableau I).

D'une manière générale, les deux tiers des effectifs disparaissent à chaque période déneigée. Dans le détail, la mortalité (+ dérive) des larves de 1 an est supérieure à celle des larves de 2 ans aux stations $\mathrm{R}$ et $\mathrm{A}$; on observe l'inverse à la station $\mathrm{B}$ où la rareté des galets limite le nombre de larves, passé une certaine taille. De même, il est possible que près de la source, l'absence d'habitat adéquat contribue à la disparition quasi complète de la cohorte âgée de 3 ans.

Les captures de larves néonates diffèrent assez largement d'une année sur l'autre en raison des possibles variations du potentiel reproducteur des populations et surtout en raison de la date plus ou moins tardive du début des éclosions qui conditionne la représentativité de l'échantillonnage. Les différences initiales s'atténuent au cours du développement et après la fonte des neiges l'effectif des cohortes de même âge ne présentent plus de différences significatives après le premier hiver dans le bas du vallon, après le deuxième hiver au-dessus de $2100 \mathrm{~m}$. Les abondances les plus variables s'observent près du névé avec des écarts de 1 à 10 pour les larves néonates et de 1 à 5 pour les cohortes de 1 an.

\section{6. - PRODUCTION LARVAIRE}

Le calcul de la production se heurte à plusieurs difficultés: 1) la longue durée de vie larvaire qui ne permet pas de suivre le développement de l'espèce de l'éclosion à l'émergence ; 2) l'hétérogénéité et le nombre réduit de relevés utilisables du fait de la sous-estimation du benthos lors des crues; 3) l'estimation de l'effectif initial (puisque durant les éclosions recrutement et mortalité sont simultanćs) et de l'effectif final (puisque les relevés ponctuels sous-estiment le dernier stade larvaire non cumulatif). Compte tenu de ces observations nous avons calculé Ia production par la méthode graphique d'Allen (1951), appliquée dans les conditions suivantes: 1) Une unique courbe de croissance-survie a été tracée d'après les résultats obtenus au cours des 3 années d'échantillonnage (fig. 6). Les valeurs de production sont donc des valeurs moyennes annuelles basées sur la production des générations qui se sont succédées durant la période d'étude. 2) Il n'est pas tenu compte des relevés non représentatifs de l'effectif réel des populations : ce sont les relevés correspondant aux périodes de crues, aux périodes d'éclosions (pour la génération néonate) et d'émergence (pour la génération mature). 3) Les effectifs néonates et matures sont déterminés par prolongement des courbes d'une part sur l'axe des ordonnées (en théorie jusqu'au point d'abscisse $P_{1}=0,00064 \mathrm{mg}$ - poids des plus petites larves récoltées, de largeur de capsule céphalique $1=0,15 \mathrm{~mm}$ ), d'autre part jusqu'au point d'abscisse $P$, correspondant au poids moyen des larves à l'émergence $\left(P_{::}=4,7 \mathrm{mg}\right.$ pour $l=3 \mathrm{~mm}$ ) ou, lorsque le développement est incomplet, au poids moyen des plus grosses larves récoltées dans le milieu.

La plus forte production s'observe à la station $\mathrm{R}(2150 \mathrm{~m})$ où les populations sont les plus abondantes (Tableau III). En aval, la crois- 

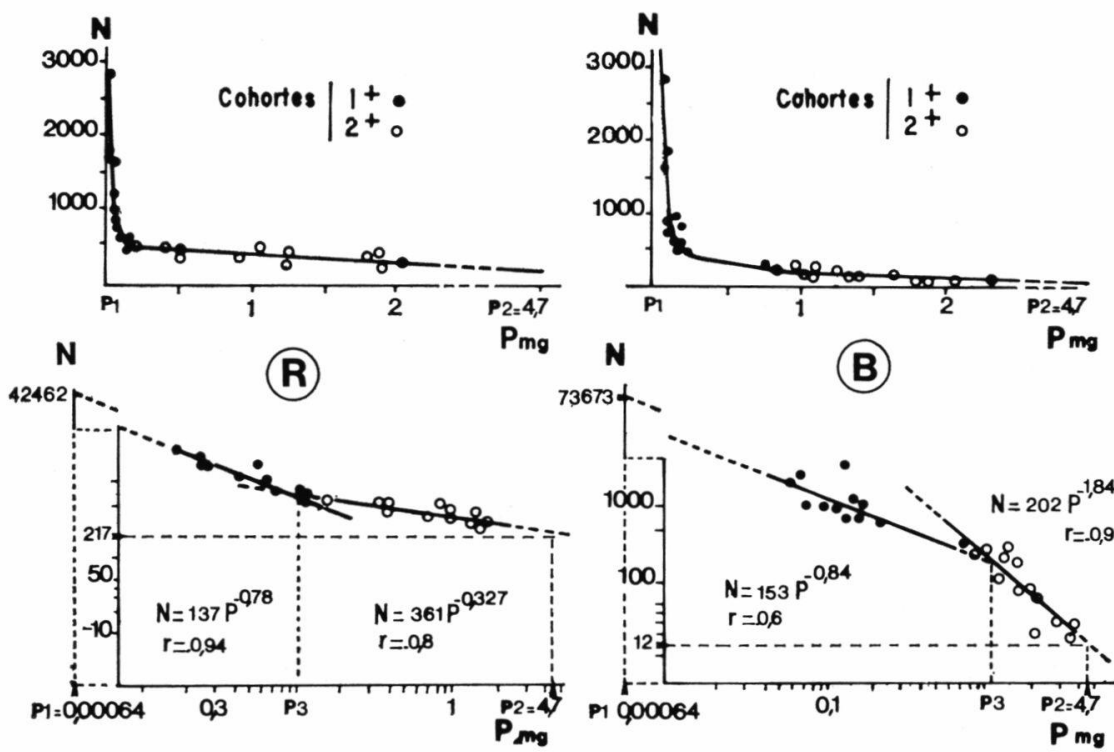

Fig. 6. - Estimation de la production - Stations R et B. En haut: courbes de croissance-survie établies sur les données brutes. En bas : courbes de croissancesurvie après transformation logarithmique des données (chaque courbe est scindée en deux portions de courbes exponentielles décroissantes dont les équations ont été calculées).

sance plus rapide et la mortalité légèrement plus faible ne compensent pas la moindre densité des effectifs. En amont, bien que les larvules soient toujours nombreuses, l'absence d'habitats propices aux larves âgées et la croissance plus lente limite la production de l'espèce.

La production s'effectue surtout durant la période déneigée. A tous les niveaux, la majeure partie est assurée par la génération devant emerger l'année suivante (génération $1^{+}$dans le cours inférieur, génération $2^{+}$dans le cours supérieur).

Les rapports $\mathrm{P} / \mathrm{B}$ établis avec, au numérateur la valeur moyenne de production annuelle et au dénominateur la biomasse moyenne présentée par' la faune au cours des périodes où le torrent est accessible (saison déneigée) varie de 2,6 (stations $\mathrm{B}$ et C) à 3,1 (station A).

Tablead III. - Production annuelle et biomasse moyenne aux diverses stations (en $\mathrm{mg}$ de poids $\mathrm{sec} / \mathrm{m}^{2}$ ).

\begin{tabular}{lrrrrr}
\hline & \multicolumn{5}{c}{ Stations } \\
\cline { 2 - 6 } & A & B & R & C & D \\
\hline Production $\left(\mathrm{mg} / \mathrm{m}^{2}\right)$ & 220 & 690 & 1485 & 675 & 375 \\
Biomasse moyenne $\left(\mathrm{mg} / \mathrm{m}^{2}\right)$ & 70 & 265 & 510 & 260 & 140 \\
\hline
\end{tabular}


Remarque. - Aux stations $\mathbf{R}$ et $\mathbf{B}$, la courbe de croissance-survie est assimilable à deux portions de courbe exponentielle décroissante qui traduisent successivement la croissance-survie des larves jeunes et âgées (fig. 6). La production peut être estimée par la valeur des intégrales calculées entre les points d'abscisse P1 et P3 d'une part, P3 et P2 d'autre part; les limites P1 et P2 correspondent aux poids des larves en début et en fin de développement déduits de la relation taille-poids. Cette méthode, basée sur le modèle décrit par Ness et Dugdale (1959) conduit à des valeurs de production supérieures d'environ $15 \%$ à celles obtenues directement de la courbe d'Allen, en raison surtout d'une estimation plus élevée des effectifs néonates.

\section{7. - DISCUSSION}

Dans la partie supérieure du torrent d'Estaragne, les populations larvaires sont toujours abondantes bien qu'une faible partie des larves seulement achève son développement. La capture de plusieurs imagos femelles venant de pondre, alors que les subimagos sont rares prouve que les migrations imaginales déià signalées par plusieurs auteurs chez les éphéméroptères (Russev 1973, Madsen et al. 1973, Thomas 1975) jouent un rôle important dans la distribution et le maintien des populations de Rhithrogena loyolaea.

Dans la partie basse du torrent, la densité du peuplement est en grande partie limitée par les amplitudes thermiques quotidiennes qui agissent vraisemblablement autant sur la vie larvaire (les quelques larves qui éclosent dans les affluents les plus chauds ne survivent pas) que sur le développement embryonnaire. A température constante, l'éclosion des œufs de Rhithrogena loyolaea se produit surtout entre 0 et $10^{\circ} \mathrm{C}$ avec un maximum entre 1,9 et $5{ }^{\circ} \mathrm{C}$ (Humpesch et Elliol.t 1980). La durée du développement embryonnaire est longue (environ 7 mois à $5{ }^{\circ} \mathrm{C}$ ) et fonction de la température. Dans le bas du réseau hydrographique, une partie des œufs subit donc au cours de l'été suivant la ponte des températures qui peuvent dépasser $10-12{ }^{\circ} \mathrm{C}$ lors des maxima diurnes. Il est possible que ces températures, même passagères, soient un facteur d'inhibition du développement embryonnaire ; elles pourraient expliquer la forte diminution de la densité de population au-dessous de $1950 \mathrm{~m}$ et la pauvreté du peuplement dans les affluents bien que le comportement des imagos tende à élargir la distribution de l'espèce. Dans la traversée de la rhodopineraie au bas du torrent, la réduction du peuplement est également liée à la nette régression du périphyton - Hydrurus et Diatomées -

Le développement de Rhithrogena loyolaea dure deux ans dans les Carpathes et la croissance larvaire est lente (Sowa 1975). Landa (1968) 
classe par contre Rhithrogena loyolaea (sub. nom. Rhithrogena tatrika Zelink - Thomas 70) parmi les espèces univoltines à croissance estivale rapide. En Estaragne, le développement larvaire dure deux ans au-dessous de $1950 \mathrm{~m}$, trois ans au-dessus de $2100 \mathrm{~m}$. Compte tenu de la durée d'incubation des œufs (Humpesch et Elliott 1980) la durée du cycle biologique entre l'oviposition et l'émergence s'étale sur 3 et 4 ans. L'allongement du cycle biologique s'effectue ici sans modification notable de la taille des individus à l'émergence.

La croissance larvaire est essentiellement fonction de la température du milieu et il existe à chaque station une bonne relation entre le taux de croissance instantané $(\mathrm{K})$ et la température (sous la forme $\mathrm{T}$ ou $\log \mathrm{T}$ ) moyenne du torrent. Les coefficients de corrélation $(r)$ voisins de 0,9 indiquent que 75 à $80 \%$ de la variation de $\mathrm{K}$ (exprimée par $\mathrm{r}^{2}-$ Snedecor et Cochran 1967, p. 176 et 177) peuvent être attribués à sa relation avec la température. La part indépendante de la température peut s'expliquer en Estaragne par l'évolution saisonnière des conditions trophiques - qualité et disponibilité de la nourriture (Brown 1961, Chapman et Demory 1963, Benech 1972, Lavandier et Pujol 1975 et par le comportement de la faune lors des fluctuations hydriques. La corrélation plus étroite qui existe entre le taux de croissance instantané $(\mathrm{K})$ des jeunes larves et le Logarithme de la température du milieu montre en outre que les variations de température ont des conséquences d'autant plus grandes sur la croissance que la température initiale est basse, ce qui favorise en particulier la rapide reprise de croissance à la fonte des neiges et réduit l'influence des températures les plus élevées.

Les taux de croissance qui varient de 0,011 à 0,04 (avec une moyenne de 0,02 ) sont toujours faibles comparés à ceux calculés pour d'autres espèces d'éphéméroptères à développement plus court (Waters 1965, Gose 1970, Pearson et Kramer 1972, Waters et Crawford 1973, Brittain 1976, Hudson et Swanson 1972 cité par Humpesch 1979); ils se rapprochent en revanche des taux de croissance d'Ephemera danica (Svensson 1977) dont le cycle biologique dure 2 ou 3 ans. Rhithrogena loyolaea apparaît donc comme une espèce sténotherme d'eau froide dont le cycle est vraisemblablement toujours long car sa croissance demeure faible dans toute la gamme de température qui caractérise son aire de répartition. En Estaragne, le développement larvaire est en outre allongé par suite de la brièveté de la période effective de croissance.

La mortalité larvaire n'est forte qu'en période déneigée, après la crue nivale, soit environ trois mois par an. De ce fait, un quart de la population survit en moyenne chaque année. Le nombre de larves néonates et matures estimé à partir des effectifs et des taux de mortalité des générations $1^{+}$et $2^{+}$conduit à un pourcentage de survie d'environ 
$12 \%$ dans le bas du torrent, de 1 à $3 \%$ dans le cours amont. Cette augmentation de la mortalité s'explique surtout par l'allongement du cycle biologique qui divise par cinq la proportion des larvules susceptibles de parvenir au terme du développement.

Ces taux de survie demeurent toutefois très forts et sont en fait certainement surestimés par suite d'une sous-évaluation du nombre de larves néonates : les effectifs des larvules déduits des nombres de larves-nymphes et du nombre d'œufs par individus (entre 3500 et 4500 , moyenne 4125 pour 12 femelles) pondéré par le pourcentage de réussite des éclosions (de l'ordre de $30 \%$, Humpesch et Elliott 1980) sont en effet dix à quinze fois plus élevés. Notons que des effectifs du même ordre sont obtenus à partir des courbes de croissance-survie établies aux stations R et B. A ces localités où, après transformation logarithmique des données, les courbes s'ajustent étroitement à un modèle mathématique simple (fig. 6) le calcul des effectifs néonates (de poids P1) et matures (de poids $\mathrm{P} 2$ ) conduit à un très faible pourcentage de survie. La survie larvaire est voisine de 5 pour 1000 à la station $R$ et tombe à 0,2 pour 1000 à la station $B$ où le cours n'offre pas d'abris suffisant aux larves âgées.

La raréfaction apparente des populations larvaires lors des crues met indirectement en évidence le déplacement de la faune vers des habitats-refuges à l'abri du courant. Ce comportement qui explique en partie la permanence et la stabilité relative du peuplement, constitue certainement un aspect fondamental de la dynamique des communautés benthiques en haute montagne (Lavandier 1979 b). La similitude des effectifs atteints chaque année par les cohortes de même âge, quelles que soient les variations initiales de recrutement, reflètent a posteriori la capacité de charge maximale $\mathrm{du}$ torrent lorsque les conditions sont les plus défavorables. L'occupation du milieu par les larves est alors favorisée par l'hétérogénéité des composantes de l'écosystème (cf. Hassel 1980) : hétérogénéité des biotopes et diversité de taille et de comportement des larves qui coexistent en permanence à différents stades de développement. Les plus importantes variations d'effectifs s'observent près de la source, où le recrutement dépend surtout de la ponte de femelles venue d'aval.

La méthode graphique d'Allen (1951) utilisée pour le calcul de la production est une méthode simple qui malgré le tracé approché des courbes donne des résultats comparables à ceux obtenus par calcul (Elliott 1973, Décamps et Lafont 1974). Sa souplesse d'application permet de traiter des données hétérogènes et les résultats, basés sur trois années d'observation, donnent certainement une bonne valeur de la capacité moyenne de production du torrent $\left(0,7 \mathrm{gr} / \mathrm{m}^{2} / \mathrm{an}\right)$. Bien que faible, cette valeur n'est pas négligeable si l'on considère que plus de 
$80 \%$ de la production annuelle s'effectue sur une période de quatre à six mois seulement.

Les rapports $\mathrm{P} / \overline{\mathrm{B}}$ dont l'intérêt est d'estimer rapidement la production à partir d'un paramètre simple, la biomasse, présentent dans tout le réseau hydrographique des valeurs voisines comprises entre 2,6 et 3,1. Ces rapports sont inférieurs aux estimations théoriques proposées par Waters (1969) et se situent parmi les valeurs faibles de la littérature (cf. Waters 1966, Gose 1970, Pearson et Kramer 1972, Sheldon 1972, Waters et Crawford 1973, Winterbourn 1974, Neveu et Lapchin 1978, Lavandier 1975, 1979 a, ...), essentiellement en raison de la coexistence de cohortes d'âges différents qui maintient en permanence une forte biomasse dans le milieu.

\section{TRAVAUX CITES}

Allen (K. R.). 1951. - The Horokowi stream. A study of a trout population. Fish. Buli. N.Z., $10: 1-231$.

Benech (V.). 1972. - Le polyvoltinisme chez Baetis rhodani Pictet (Insecta, Ephemeroptera) dans un ruisseau à Truites des Pyrénées-Atlantiques, Le Lissuraga. Ann. Hydrobiol., 3 (2) : 141-171.

Brittain (J. E.). 1976. - Experimental studies on nymphal growth in Leptophlebia vespertina (L.) (Ephemeroptera). Freshwater Biol., 6 (5) : 445-449.

Brown (D. S.). 1961. - The food of the larvae of Cloeon dipterum L. and Baetis rhodani (Pictet) (Insecta, Ephemeroptera). J. Anim. Ecol., $30:$ 55-75.

Chapman (D. W.) and Demory (R.). 1963. - Seasonal changes in the food myested by aquatic insect larvae and nymphs in two Oregon streams. Ecology, 44 : 140-146.

Decamps (H.) et Lafont (M.). 1974. - Cycles vitaux et production des Micrasema pyrénéennes dans les mousses d'eau courante (Trichoptera, Brachycentridae). Annls Limnol., 10 (1) : 1-32.

Elliotr (J. M.). 1973. - The life cycle and production of the leech Erpobdella octoculata (L.) (Hirudinea: Erpobdellidae) in a lake district stream. $J$. Anim. Ecol., 42: 435-448.

Elliotr (J. M.). 1978. - Some methods for the statistical analysis of samples of benthic invertebrates. Scient. Publs Freshwat. Biol. Ass., $25: 160$ p.

Gose (K.). 1970. - Life history and production rates of Ephemera strigata (Epheroptera). Jap. J. Limnol., $31: 21-26$.

Hamilton (A. L.). 1969. - On estimating annual production. Limnol. Oceanog., 4 : 771-78:

Hassel (M. P.). 1980. - Some consequences of habitat heterogeneity for population dynamics. Oikos, $35: 150-160$.

Humpesch (U. H.). 1979. - Lite cycles and growth rates of Baetis spp. (Ephemeroptera: Baetidae) in the laboratory and in two stony streams in Austria. Freshwater Biol., 9 (5) : 467-479.

HuMPESCH (U. H.) and ElliotT (J. M.). 1980. - Effect of temperature on the hatching time of eggs of three Rhithrogena spp. (Ephemeroptera) from austrian streams and an english stream river. J. Anim. Ecol., 49 (2) : 643-661.

LANDA (V.). 1968. - Developmental cycles of central european ephemeroptera and their interrelations. Acta entomologica bohemoslovaca, 65 : 276-284. 
LaVANdier (P.). 1974. - Ecologie d'un torrent pyrénéen de haute montagne. I. Caractéristiques physiques. Annls Limnol,, 10 (2) : 173-219.

Lavandier (P.). 1975. - Cycle biologique et production de Capnioneura brachyptera D. (Plécoptères) dans un ruisseau d'altitude des Pyrénées centrales. Annls Limnol., 11 (2) : 145-156.

LAVANDiER (P.). 1979 a. - Cycle biologique, régime alimentaire, production d'Arcynopteryx compacta (Plecoptera, Perlodidae) dans un torrent de haute altitude. Bull. Soc. Hist. nat. Toulouse, $115:$ 140-150.

Lavandier (P.). $1979 \mathrm{~b}$. - Ecologie d'un torrent pyrénéen de haute montagne: l'Estaragne. Thèse sciences Université Paul-Sabatier Toulouse: $532 \mathrm{p}$.

Lavandier (P.) et Pujol (J. Y.). 1975. - Cycle biologique de Drusus rectus (Trichoptera) dans les Pyrénées centrales: influence de la température et de l'enneignement. Annls Limnol., 11 (3) : 255-262.

Madsen (B. L.), Bengtson (J.) et Butz (I.). 1973. - Observations on upstream migration by imagines of some Plecoptera and Ephemeroptera. Limnol. Oceanog., 18 (4) : 678-681.

Ness (J.) et Dugdale (R. C.). 1959. - Computation of production for populations of aquatic midge larvae. Ecology, 40: 425-430.

Neveu (A.) et Lapchiv (L.). 1978. - Ecologie des principaux invertébrés filtreurs de la basse Nivelle (Pyrénées atlantiques). I. Simuliidae (Diptera, Nematocera). Annls Limnol., 14 (3) : 225-244.

Pearson (W. D.) and Kramer (R. H.). 1972. - Drift and production of two aquatic insects in a moutain stream. Ecol. Monog., 42 : 365-385.

Russev (B.). 1973. - Kompensation flug bei der Ordnung Ephemeroptera. Proc. First Int. Conf. Ephemeroptera, Brill (Leiden) : 132-142.

Sheldon (A. L.). 1972. - Comparative ecology of Arcynopteryx and Diura (Plecoptera) in a California stream. Arch. Hydrobiol., 69: 521-546.

Snedecor (G. W.) et Cochran (W. G.). 1967. - Statistical methods. Ames, lowa: 593 p.

SowA (R.). 1975. - Ecology and biogeography of mayflies (Ephemeroptera) of running waters in the Polish part of the Carpathians. I. Distribution and quantitative analysis. II. Life cycles. Acta Hydrobiologica, 17: 223-297 and 319-353.

SvensSON (B.). 1977. - Life cycle, energy fluctuations and sexual differentation in Ephemera danica (Ephemeroptera), a stream-living mayfly (Ephemeridae). Oikos, 29 (1) : 78-86.

Thomas (A.). 1970. - Sur la taxonomie de deux Rhithrogena des Pyrénées (Ephemeroptera, Heptageniidae). Annls Limnol., 6 (3) : 305-315.

Thomas (A. G. B.). 1975. - Ephéméroptères du Sud-Ouest de la France. I. Migrations d'imagos à haute altitude. Annls Limnol., 11 (1) : 47-66.

Waters (T. F.). 1966. - Production rate, nopulation density, and drift of a stream invertebrate. Ecology, 47 (4) : 595-604.

WATERS (T. F.). 1969. - The turnover ratio in production ecology of freshwater invertebrates. Amer. Nat., $103:$ 173-185.

Waters (T.F.) et CRAWford (G.W.). 1973. - Annual production of a stream mayfly population: comparison of methods. Limnol. Oceanog., 18 : 286-296.

WinterbouRn (M. J.). 1974. - The life histories, trophic relations and production of Stenoperla prasina (Plecoptera) and Delatidium sp. (Ephemeroptera) in a New Zealand river. Freshwater Biology, $4:$ 507-524. 